\title{
The Role of Beta-Frequency Neural Oscillations in Motor Control
}

\author{
Nick J. Davis, ${ }^{1}$ Simon P. Tomlinson, ${ }^{2}$ and Helen M. Morgan ${ }^{1}$ \\ ${ }^{1}$ School of Psychology and ${ }^{2}$ Graduate Programme in Psychology, School of Psychology, Bangor University, Bangor LL57 2AS, United Kingdom \\ Review of Feurra et al.
}

Human sensorimotor and cognitive behavior is associated with changes in the oscillatory activity of the brain. For example, the integration of diverse aspects of a stimulus into a unitary percept is related to synchronized oscillations in the gamma range $(30-100 \mathrm{~Hz})$, while power in the alpha band $(8-12 \mathrm{~Hz})$ increases during relaxation. Motor activity is associated with changes in beta frequency oscillations, which has a range of $15-30 \mathrm{~Hz}$ and peaks at $\sim 20 \mathrm{~Hz}$. Voluntary movement is associated with a drop in power (desynchronization) in this frequency range, and the termination of movement is followed by a restoration of power (Salmelin and Hari, 1994). One hypothesis is that beta activity represents the status quo (Engel and Fries, 2010). Parkinson's disease, in which sufferers find it difficult to initiate or change movements, is notably associated with higher levels of beta synchrony (Schnitzler and Gross, 2005), suggesting that the enhanced beta activity is preventing change from the status quo.

The recently developed technique of transcranial alternating current stimulation (tACS) may be a way to investigate

Received Oct. 10, 2011; revised Nov. 7, 2011; accepted Nov. 10, 2011.

This work was supported by an EU FP7 Grant under the Future and Emerging Technologies programme: FET-Open 222079 (HIVE) (to N.J.D. and H.M.M.). We are grateful to Dr. Martyn Bracewell for helpful comments.

N.J.D. and H.M.M. are supported by EU FP7 Grant FET-Open 222079 (HIVE) to Dr. Martyn Bracewell. We are grateful to Dr. Bracewell for helpful comments.

Correspondence should be addressed to Dr. Nick Davis at the above address. E-mail:n.davis@bangor.ac.uk.

DOI:10.1523/JNEUROSCI.5106-11.2012

Copyright $\odot 2012$ the authors $\quad 0270-6474 / 12 / 320403-02 \$ 15.00 / 0$ the role of oscillatory fields in brain function. In tACS, two electrodes are placed on the head and an alternating current is passed between them. The induces an oscillatory electrical field across the brain between the two electrodes. This is likely to induce neural synchronization at the frequency of tACS in the cortical areas underneath the electrodes, although relatively little is known of the electrophysiological effect of tACS on the brain (Zaghi et al., 2010). Compared with other brain stimulation techniques, such as transcranial magnetic stimulation (TMS) or direct transcranial current stimulation (tDCS), tACS has a number of advantages. The effect of the field is short-lived, in that no effect of tACS is evident after the current is removed, whereas the effects of $\mathrm{tDCS}$ outlast stimulation by several minutes. The stimulation is also usually not perceptible to the participant, whereas $\mathrm{tDCS}$ may prickle the skin and TMS involves an audible click.

In a recent paper in The Journal of $\mathrm{Neu}$ roscience, Feurra and colleagues (2011) applied tACS at four different frequencies to the primary motor cortex of human volunteers. The effect of tACS on the motor cortex was determined by using single pulses of TMS over the hand area of left motor cortex to generate muscular responses measured in the participants' right hand [motor-evoked potentials (MEPs)]. The amplitude of the MEP is thought to relate to the excitability of the corticospinal pathway. MEP size is frequently taken as a measure of the preparedness of the motor system for action, for example when observing actions performed by another agent (Fadiga et al., 2005). Feurra and colleagues (2011) used four frequencies of tACS: $20 \mathrm{~Hz}$, and control frequencies of 5, 10, and $40 \mathrm{~Hz}$ (representing the theta, alpha, and gamma ranges, respectively). In addition, a control site of right parietal cortex was stimulated to check for spatial specificity of tACS stimulation. Feurra et al. (2011) found that tACS stimulation at all frequencies had an enhancing effect on MEPs (their Fig. 2), although pairwise tests among the conditions showed that only the target frequency of $20 \mathrm{~Hz}$ had a statistically significant enhancement compared with baseline and compared with the other stimulation conditions. These other stimulation conditions included the parietal stimulation site. In a further control experiment, no effects of stimulation frequency on MEP size were detected when MEPs were generated by applying TMS to the ulnar nerve of the right arm, showing that the effects were not due to a physical interaction between the tACS-induced current and the current generated by TMS. The results of Feurra and colleagues' (2011) experiment suggest that beta-frequency tACS can affect motor cortical excitability. These effects were spatially specific, since stimulating the contralateral parietal cortex did not affect MEP size, and frequency specific, since tACS at different frequencies did not change MEP size either.

The findings of this work are interesting because there is no a priori reason to 
believe that injecting a current at a similar frequency to one that is naturally present would have the same effect as the naturally occurring rhythm. At present, there is no known functional role for oscillations in the beta frequency, so it is not clear whether beta activity encodes a specific quality of motor activity or planning or is simply an epiphenomenon. If activity in the beta band were an epiphenomenon, we would not expect there to be any effect of adding power in this frequency. Conversely if activity in this range were functionally and causally related to motor behavior in itself, regardless of what population of neurons were participating in the rhythm, it would suggest that motor information is carried by neural synchronization; in this case, the substantial additional current added by the tACS would be likely to interfere with the ongoing processing in the cortex. In any case, it is likely that the exact frequency that best represents the beta band is specific to a person and cortical area. For example, in one study, hand and foot movements were associated with different beta frequencies over the corresponding areas of sensorimotor cortex, and within each area the peak frequency varied across individuals (Neuper and Pfurtscheller, 2001). Therefore using a single frequency for all participants in a study could mean that some people would be less likely to show an effect. It is notable that in the study of Feurra et al. (2011), there were four subjects (of 15) who responded less well to 20 $\mathrm{Hz}$ than to other frequencies of tACS, although in these four subjects the beta stimulation gave the second-best effect. The individual variation in peak beta frequency was not measured in that study.

Feurra et al. (2011) report an enhancement in MEP size, which is usually taken to represent a positive change in motor function. Yet a recent study that used 20 $\mathrm{Hz}$ tACS to left motor cortex showed a detrimental effect on motor behavior in the right upper limb: during tACS, people's movements became slower (Pogosyan et al., 2009). How can we reconcile the results of Feurra et al. (2011) and of Pogosyan et al. (2009)? We suggest that enhancing oscillatory activity through the use of tACS raises the excitability of the whole motor cortex (hence the raised MEP amplitudes); however, it does so nonselectively. Given that primary motor cortex is functionally organized into synergies (Holdefer and Miller, 2002), coactivation of a number of synergy clusters is likely to have a deleterious effect on any one action. Put this way, synchronizing oscillatory activity of neurons in motor cortex could be both physiologically enhancing and functionally detrimental. This is consistent with previous work showing that movement disorders such as Parkinson's disease are characterized by synchronization in the beta band.

Modulating brain activity through noninvasive current stimulation is a new tool in cognitive neuroscience and has already produced interesting results. The recent work by Feurra et al. (2011) is an important step in understanding the physiological events that occur during tACS. However, the work also shows the causal role that beta-frequency oscillatory brain activity plays in motor behavior: if beta activity were an epiphenomenon, adding more activity would not change the state of the motor system. The work also raises a number of questions: How close does the stimulating frequency need to be to the participant's individual beta peak? What is the somatotopic specificity of the effect (would leg MEPs be less af- fected than hand MEPs)? Can the current distribution be shaped to stimulate a smaller area of the cortex, leading to a more somatotopically specific effect? Can altering the stimulation parameters generate both enhancing and impairing motor outcomes? tACS is a new tool in cognitive neuroscience and carefully controlled studies of this kind hold great promise for future studies of the neural control of behavior.

\section{References}

Engel AK, Fries P (2010) Beta-band oscillationssignalling the status quo? Curr Opin Neurobiol 20:156-165.

Fadiga L, Craighero L, Olivier E (2005) Human motor cortex excitability during the perception of others' action. Curr Opin Neurobiol 15:213-218.

Feurra M, Bianco G, Santarnecchi E, Del Testa M, Rossi A, Rossi S (2011) Frequency-dependent tuning of the human motor system induced by transcranial oscillatory potentials. J Neurosci 31:12165-12170.

Holdefer RN, Miller LE (2002) Primary motor cortical neurons encode functional muscle synergies. Exp Brain Res 146:233-243.

Neuper C, Pfurtscheller G (2001) Evidence for distinct beta resonance frequencies in human EEG related to specific sensorimotor cortical areas. Clin Neurophysiol 112:2084-2097.

Pogosyan A, Gaynor LD, Eusebio A, Brown P (2009) Boosting cortical activity at beta-band frequencies slows movement in humans. Curr Biol 19:1637-1641.

Salmelin R, Hari R (1994) Characterization of spontaneous MEG rhythms in healthy adults. Electroencephalogr Clin Neurophysiol 91:237248.

Schnitzler A, Gross J (2005) Normal and pathological oscillatory communication in the brain. Nat Rev Neurosci 6:285-296.

Zaghi S, Acar M, Hultgren B, Boggio PS, Fregni F (2010) Noninvasive brain stimulation with low-intensity electrical currents: putative mechanisms of action for direct and alternating current stimulation. Neuroscientist 16: 285-307. 